Conversely, SDIL proponents demonstrated three sources of inconsistency: 1) change in ideological stance; 2) pursuit of academic rigour; and 3) inconsistent arguments.

Discussion Public health policy advocates engaged in media debates are faced with the direct lobbying and denialism tactics of producers and marketers of unhealthy commodities. These advocates may benefit from increasing awareness of typical UCI tactics, presenting clear and consistent objectives, and supporting arguments with quality evidence. Our CDA contributes to a growing body of literature concerning media debates about upstream legislative public health measures focussing on unhealthy commodities.

\section{OP3 COST-EFFECTIVENESS OF THE U.S. FDA ADDED SUGAR LABELING POLICY FOR IMPROVING CARDIOMETABOLIC HEALTH: MICROSIMULATION MODELLING STUDY}

${ }^{1} \mathrm{C}$ Kypridemos*, ${ }^{2} Y$ Huang, ${ }^{2} \mathrm{~J} \mathrm{Liu,}{ }^{2} \mathrm{Y}$ Lee, ${ }^{1} \mathrm{~J}$ Pearson-Stuttard, ${ }^{1} \mathrm{~B}$ Collins, ${ }^{1} \mathrm{P}$ Bandosz, ${ }^{1} \mathrm{~S}$ Capewell, ${ }^{1} \mathrm{M}$ O'Flaherty, ${ }^{2} \mathrm{R}$ Micha, ${ }^{3} \mathrm{~L}$ Whitsel, ${ }^{2} \mathrm{P}$ Wilde, ${ }^{2} \mathrm{D}$ Mozaffarian. ${ }^{1}$ Department of Public Health and Policy, University of Liverpool, Liverpool, UK; ${ }^{2}$ Friedman School of Nutrition Science and Policy, Tufts University, Boston, Massachusetts, USA; ${ }^{3}$ American Heart Association, Washington DC, USA

\subsection{6/jech-2018-SSMabstracts.3}

Background Excess added sugars, particularly from sugarsweetened beverages (SSBs), are associated with cardiometabolic risk including type 2 diabetes (T2D) and cardiovascular disease (CVD). Despite recent declines in SSB intake in the US, added sugar intake from SSBs and foods remains high and substantially exceeds dietary recommendations. The US Food and Drug Administration (FDA) announced major changes to the Nutrition Facts panel in 2016, including mandatory labeling of added sugar content, as a strategy to target added sugars in packaged foods and beverages. However, the potential health and economic benefits of the FDA proposal remain unclear.

Objective To estimate the cardiometabolic and economic effects of implementing FDA's added sugar labeling policy from 2018-2037.

Methods We used the validated, US IMPACT Food Policy microsimulation model to estimate the T2D and CVD cases averted and quality-adjusted life-years (QALYs) gained from the FDA policy for US adults age 30-84 years.

We modelled two policy scenarios:

1. implementation of the FDA added sugar labeling policy (sugar label)

2. anticipated reactive industry reformulation (sugar label + reformulation).

Model inputs included national health statistics from CDC; added sugar intakes from NHANES; policy effects on consumer intake from labeling intervention studies; obesity-mediated and direct effects of added sugars from SSBs and other foods on T2D and CVD from meta-analyses; policy costs including government administrative costs; industry compliance costs from federal government reports; healthcare costs including medical, productivity, and indirect costs from the AHA and American Diabetes Association. All costs were inflated to constant 2017 US dollars, then discounted annually at 3\%. We took a societal perspective and assumed a willingness to pay of $\$ 100000$ per QALY. Probabilistic sensitivity analysis accounted for model parameter uncertainty and population heterogeneity.
Results Between 2018 and 2037, the sugar label could prevent approximately 354,400 CVD cases $(95 \%$ UIs: 167,000673,500) and 599300 diabetes cases (302,400-957,400), gain some 727,000 QALYs (401,300-1,138,000), and save approximately $\$ 31$ bn (15.7-54.5) in net costs from a healthcare perspective or $\$ 62$ bn (33.1-103.3) from a societal perspective.

For the sugar label+reformulation scenario, corresponding gains could be substantially larger: approximately 708,800 CVD cases $(369,200-1,252,000), 1.2 \mathrm{~m}$ diabetes cases (0.7$1.7)$, and $1.3 \mathrm{~m}(0.8-1.9)$ QALYs, with some $\$ 58$ bn (31.992.4) and $\$ 113$ bn (67.3-175.2) net savings from the healthcare and societal perspectives respectively. Scenarios were estimated with $>80 \%$ probability to be cost-saving by 2023 .

Conclusion Implementing the FDA added sugar labeling policy could generate substantial health gains and cost savings for the US population.

\section{OP4 ASSESSING THE IMPACT OF THE BARBADOS SUGAR SWEETENED BEVERAGE TAX ON GROCERY STORE BEVERAGE SALES AND NATIONAL IMPORTS: AN INTERRUPTED TIME SERIES ANALYSIS}

\footnotetext{
1,2 MA Alvarado* ${ }^{1,2} \mathrm{~N}$ Unwin, ${ }^{1} \mathrm{~S}$ Sharp, ${ }^{2}$ Hambleton, ${ }^{2} \mathrm{MM}$ Murphy, ${ }^{2} \mathrm{TA}$ Samuels, ${ }^{3} \mathrm{M}$ Suhrcke, ${ }^{1} \mathrm{~J}$ Adams, on behalf of the Barbados SSB Tax Evaluation Group. ${ }^{1}$ Centre for Diet and Activity Research, MRC Epidemiology Unit, University of Cambridge, Cambridge, UK; ${ }^{2}$ George Alleyne Chronic Disease Research Centre, Caribbean Institute for Health Research, University of the West Indies, Bridgetown, Barbados; ${ }^{3}$ Centre for Health Economics, University of York, UK
}

\subsection{6/jech-2018-SSMabstracts.4}

Background A 10\% tax on sugar sweetened beverages (SSBs) was implemented in Barbados in September 2015. This study aims to assess whether sales of SSBs and non-SSBs changed following implementation of the tax.

Methods We used electronic sales data from a major grocery chain from January 2012 - January 2017, and national import data from the Barbados Statistical Service from January 2013July 2017. Grocery store data were used to calculate litres sold per week of SSBs, non-SSBs, and beverage sub-categories (sodas, waters) over 264 weeks. We used an interrupted time series (ITS) design to assess whether the introduction of the tax was associated with a change in level and trend in sales. All analyses controlled for inflation, seasonality and tourism.

Using national import data, we calculated cumulative litres imported (to account for stockpiling) by beverage category. We assessed whether the introduction of the tax was associated with a change in the trend of the cumulative litres imported. To enable comparison, we re-estimated the grocery store models separately for locally manufactured and imported beverages. STATA/SE 14.2 was used for all analyses.

Results In 2016 (the first full year of tax implementation), the average decrease in SSB grocery store sales was $-2,822$ litres/ week [95\% CI $-6,397$ to 752 ] compared to the estimated counterfactual without the tax. The change per week increased over time, with an average decrease from July 2016-December 2016 of $-4,349$ litres/week [95\% CI $-8,307$ to -391$]$. Locally manufactured SSBs decreased by an average of $-5,814$ litres/week [95\% CI $-8,692$ to -2,936] and imported SSBs increased by an average 4277 litres/week [95\% CI 2,872 to 5682]. Non-SSB sales increased by 1954 litres/week [95\% CI 507 to 3401], with water accounting for an average increase of 1435 litres/week [95\% CI 393 to 2477]. 
Monthly SSB imports increased by 369000 litres per month following the tax [95\% CI 325,000 to 413 000]. Non-SSB imports increased by 281000 litres per month [95\% CI 268,000 to 294, 000].

Conclusion The Barbados SSB tax was not associated with a reduction in SSB sales at this grocery store chain, although there is evidence of a reduction at the end of the study period. National data suggest that SSB imports (which may be cheaper) increased. The grocery store analysis also suggest that sales of imported SSBs increased, while locally manufactured SSBs decreased, suggesting that consumers may be substituting to imported beverages. A higher rate of tax may be needed to reduce consumption of SSBs.

\section{OP5 \#EXPLORING INDUSTRY INFLUENCE ACROSS ALCOHOL AND SUGAR PRICING POLICIES IN THE UK: A DISCOURSE NETWORK ANALYSIS}

S Hilton*, C Buckton, G Fergie. MRC/CSO SPHSU, University of Glasgow, Glasgow, UK

\subsection{6/jech-2018-SSMabstracts.5}

Background Increasingly unhealthy commodity industries (UCIs) strategically use the news media to influence public opinion and the political agenda in favour of advancing their preferred policy options. In politically-charged pricing policy debates, such as Minimum Unit Pricing (MUP) for alcohol and sugar-sweetened beverage (SSB) taxation industry efforts to disrupt the introduction of these pricing policies has been significant. By comparing MUP for alcohol and SSB taxation media debates using discourse network analysis (DNA) we aim to visually map the actors and their relationships highlighting similarities and differences across industry sectors.

Methods Eleven national UK newspapers, spanning political views and genre, were searched using the Nexis database between May 2011 and November 2012 to identify all published articles relating to alcohol and pricing, and between May 2015 and November 2016 to identify all published articles relating to sugar/beverage and tax/levy. Statements made by actors and organisations in the debates were inductively identified and coded using network analysis software to produce relational data to generate visualisations of discourse networks.

Results For MUP for alcohol 1435 statements made by 151 individuals from 87 organisations were coded in 351 articles. For SSB taxation 3882 statements made by 214 individuals from 177 organisations were coded in 511 articles. The construction of MUP for alcohol and SSB taxation networks provides the first visual evidence of the positioning of industry representatives across two policy debates.Both networks show tight discourse coalitions of manufactures acting in opposition to policy advocates, with the largest corporations most active, and most central in these coalitions at key points in the debate. Less active industry representatives and cross-sector corporations (such as supermarkets) are more peripheral to the network, indicating both cleavages within industries and across corporate actors.

Conclusion By comparing the discourse networks across two highly contested pricing policy debates, we have visualised the complex network of actors and relationships operating to directly influence pricing policy-making via the media. Conducting comparative discourse network analysis across policy debates shows promise for better understanding the common tactics of different UCIs to disrupt public health policies. This is important for supporting public health advocates to develop more effective media advocacy strategies for exposing and opposing UCI tactics and strategies and in identifying public health messages which might be targeted to generate public and policy support for pricing policies.

\section{Obesity}

\section{OP6 RAPID WEIGHT GAIN IN THE FIRST FEW YEARS OF LIFE AND CHILD-TO-ADOLESCENCE BMI TRAJECTORIES: THE UK MILLENNIUM COHORT STUDY}

${ }^{1} Y L_{L}{ }^{*},{ }^{2} \mathrm{~A}$ Pearce, ${ }^{1} \mathrm{~L} \mathrm{Li} .{ }^{1}$ GOS Institute of Child Health, UCL, London, UK: ${ }^{2} \mathrm{MRC} / \mathrm{CSO}$ Social and Public Health Sciences Unit, University of Glasgow, Glasgow, UK

\subsection{6/jech-2018-SSMabstracts.6}

Background High birthweight and rapid weight gain (RWG) in early life are independently and positively associated with obesity in childhood and adulthood. It is unclear whether RWG can affect BMI growth from childhood to adolescence. RWG is common among low-birth-weight infants, especially following intrauterine growth retardation. However, whether the association between RWG and BMI trajectory differs by birthweight groups is not well understood. We aimed to investigate the effect of RWG (birth-3 y) on BMI trajectory (5-14 y) and whether associations differed by birthweight groups.

Methods We used data from the Millennium Cohort Study, a representative sample of children born in the UK in 2000-2002. We included term singletons with available information on weight gain (birth-3 y) and $1+$ BMI measurement between $5 \mathrm{y}$ and $14 \mathrm{y}(\mathrm{n}=11,522)$. Weight at birth and $3 \mathrm{y}$ were converted to internal standard deviations scores (SDS). RWG was defined as change in weight SDS from birth to $3 \mathrm{y}>0.67 \mathrm{SDS}$, which is commonly used and represents the width of each percentile band on standard growth charts. Mixed effects fractional polynomial models were applied to estimate the effects of RWG on BMI trajectories, for boys and girls separately, before and after adjustment for maternal pre-pregnancy, maternal smoking during pregnancy, parity, ethnicity, breastfeeding, early introduction to solid foods, maternal education, and family income. The analysis was further stratified by low birthweight (LBW) status.

Results Boys and girls who experienced RWG were heavier than their non-RWG counterparts at $5 \mathrm{y}$ by $1.21 \mathrm{~kg} / \mathrm{m}^{2}$ [95\% CI 1.10 to 1.33 ] and $1.25 \mathrm{~kg} / \mathrm{m}^{2}$ [1.13-1.38] respectively; continued to gain weight more rapidly between $5 \mathrm{y}$ and $14 \mathrm{y}$, especially in childhood $\left(5-7\right.$ y) by $0.2-0.3 \mathrm{~kg} / \mathrm{m}^{2}$ per year. These differences persisted after adjustment for potential confounders. The effect of RWG on BMI was particularly higher among non-LWB children, whose estimated BMI trajectories exceeded the International Obesity Task Force overweight reference lines. For example, at $14 \mathrm{y}$ the estimated mean BMI was $24.41 \mathrm{~kg} / \mathrm{m}^{2}$ [23.91-24.45] for non-LBW RWG girls (vs. $22.07 \mathrm{~kg} / \mathrm{m}^{2}$ for LWB RWG girls), exceeding the gender- and age-specific cut-off point of $23.34 \mathrm{~kg} / \mathrm{m}^{2}$ for overweight. Sensitivity analysis using 3-category birthweight variable (low/normal/high) showed that the trajectories of high-birthweight children were similar to those of normalbirthweight children. 